\title{
Síndrome de quebra-nozes: o que há de novo?
}

\section{Nutcracker syndrome: what's new?}

\section{Mateus de Sousa Rodrigues ${ }^{1}$, Manoel Pereira Guimarães ${ }^{1}$, Leonardo Fernandes e Santana ${ }^{1}$, Karyne Krysley Almeida Coelho" ${ }^{1}$, Vinícius Alves Santos ${ }^{1}$, Américo Alves da Mota Júnior², Orlando Vieira Gomes ${ }^{1,2}$}

\author{
${ }^{1}$ Universidade Federal do Vale do São Francisco (UNIVASF) \\ ${ }^{2}$ Hospital Universitário da Universidade Federal do Vale do São Francisco (HU-UNIVASF)
}

Autor correspondente: manoelpeguimaraes@ gmail.com

Artigo recebido em 16/10/2020 e aceito em 04/12/2020

\begin{abstract}
RESUMO
A síndrome do quebra-nozes ocorre devido à compressão extrínseca da veia renal esquerda entre a aorta e a artéria mesentérica superior. Apesar da relevância clínica dessa síndrome, existem poucas publicações brasileiras sobre o tema, e o subdiagnóstico do quadro é frequente. O objetivo foi de realizar uma revisão bibliográfica sobre a síndrome de quebranozes. Trata-se de uma revisão de literatura a partir dos artigos publicados nos últimos 47 anos nas seguintes bases de dados: Scholar Google, SciELO, Pubmed Central®, ScienceDirect. A síndrome do quebra-nozes possui manifestações diversas, sendo as mais comuns dores no flanco esquerdo e hematúria. O diagnóstico é difícil e pode ser, muitas vezes, tardio. Existem várias opções de tratamento, sendo o procedimento cirúrgico aberto o mais realizado, e, destes, a transposição de veia renal esquerda tem sido a abordagem cirúrgica mais empregada e mais eficaz no tratamento da síndrome. Uma alternativa menos invasiva ao tratamento cirúrgico aberto que vem ganhando espaço é a abordagem endovascular, apesar de algumas complicações terem sido relatadas. A síndrome de quebra-nozes é um quadro clínico de difícil diagnóstico, é um importante diagnóstico diferencial de hematúria, requer alta suspeição clínica e é potencialmente tratável. Desta forma, este artigo ampliará a literatura científica sobre a temática no sentido contribuir para a redução dos subdiagnósticos da síndrome.
\end{abstract}

Palavras-chave: Síndrome de quebra-nozes; epidemiologia; diagnóstico; tratamento.

\begin{abstract}
The nutcracker syndrome occurs due to extrinsic compression of the left renal vein between the aorta and the superior mesenteric artery. Despite the clinical relevance of this syndrome, there are few Brazilian publications on the subject, and underdiagnosis of the condition is frequent. The objective was to perform a bibliographical review on the nutcracker syndrome. This is a literature review from articles published in the last 47 years in the following databases: Scholar Google, SciELO, Pubmed Central®, ScienceDirect. Nutcracker syndrome has several manifestations, with the most common being pain in the left flank and hematuria. The diagnosis is difficult and can be often late. There are several treatment options, with open surgical procedure being the most accomplished, and of these, left renal vein transposition has been the most used and effective surgical approach in the treatment of the syndrome. A less invasive alternative to open surgical treatment that is gaining space is the endovascular approach, although some complications have been reported. Nutcracker syndrome is clinically difficult to diagnose, is na important differential diagnosis of hematuria, requires high clinical suspicion and is potentially treatable. Thus, this article will expand the scientific literature on the subject in order to contribute to the reduction of underdiagnosis of the syndrome.

Keywords: Nutcracker syndrome; Epidemiology; Diagnosis; Treatment.
\end{abstract}




\section{INTRODUÇÃO}

A síndrome do quebra-nozes ocorre devido à compressão extrínseca da veia renal esquerda (VRE) entre a aorta e a artéria mesentérica superior (AMS) ${ }^{1}$. Em 1944, Grant descreveu pela primeira vez a compressão da VRE, encontrando a anatomia análoga a uma porca em quebra-nozes $^{2}$. Chait et al. utilizou pela primeira vez o termo quebra-nozes ${ }^{3}$, apesar de frequentemente ainda ser creditado ao Schepper ${ }^{4}$. Deve ser suspeitada quando ocorre distensão da VRE com estreitamento abrupto à medida que passa posterior à $\mathrm{AMS}^{5}$. Esta entidade clínica leva a hipertensão venosa renal ${ }^{6}$ e/ou veia ovariana ${ }^{7}$. A apresentação clínica inclui hematúria, proteinúria, dor de flanco esquerdo, varicocele em homens, varizes vulvares em mulheres, disúria, intolerância ortostática, fadiga e congestão pélvica ${ }^{7-9}$, ocorrendo mais frequentemente em mulheres ${ }^{8,10-13}$. O tratamento da síndrome geralmente é realizado por meio de procedimento cirúrgico aberto ou de métodos endovasculares ${ }^{7,14}$.

Apesar da relevância clínica dessa síndrome, existem poucas publicações brasileiras sobre o tema, e o subdiagnóstico do quadro é frequente. Nesse sentido, o objetivo desse trabalho é realizar uma revisão bibliográfica sobre a síndrome de quebra-nozes.

\section{MATERIAL E MÉTODOS}

Trata-se de uma revisão de literatura a partir dos artigos publicados nos últimos 47 anos nas seguintes bases de dados: Google Scholar, SciELO, Pubmed Central ${ }^{\circledR}$ (PMC) e ScienceDirect. Foram consultados 45 artigos de diferentes autores nessas plataformas (Tabela 1). Os termos utilizados na pesquisa dos artigos nessas bases de dados foram: 'nutcracker syndrome', 'pathophysiology nutcracker syndrome', 'epidemiology nutcracker syndrome', 'clinical manifestations of nutcracker syndrome', 'diagnosis of nutcracker syndrome', 'treatment of nutcracker syndrome'.
Tabela 1: características dos estudos

\begin{tabular}{|c|c|c|}
\hline Autor & Ano & $\begin{array}{c}\text { Fator de impacto do } \\
\text { Periódico }\end{array}$ \\
\hline Chait et al. & 1971 & 1,32 \\
\hline Wendel et al. & 1980 & 5,64 \\
\hline Zerhouni et al. & 1980 & 5,64 \\
\hline Beinart $\mathrm{C}$ & 1982 & 5,64 \\
\hline Stewart, Reiman & 1982 & 1,86 \\
\hline Nishimura et al. & 1986 & 7,60 \\
\hline Hohenfelliner et al. & 1991 & 5,64 \\
\hline Shokeir et al. & 1994 & 4,68 \\
\hline Stavros et al. & 1994 & 1,71 \\
\hline Neste et al. & 1996 & 2,75 \\
\hline Chuang et al. & 1997 & 5,64 \\
\hline Park et al. & 2000 & 4,19 \\
\hline Scultetus et al. & 2001 & 3,24 \\
\hline Hohenfellner et al. & 2002 & 1,86 \\
\hline Hosotani et al. & 2003 & 1,81 \\
\hline Zhou et al. & 2003 & 5,64 \\
\hline Shin et al. & 2005 & 4,19 \\
\hline Kim et al. & 2005 & 2,75 \\
\hline Ahmed et al. & 2006 & 3,64 \\
\hline Rudloff et al. & 2006 & 1,17 \\
\hline Correia et al. & 2007 & 0,13 \\
\hline Zhang et al. & 2007 & 1,17 \\
\hline Chen et al. & 2009 & 1,59 \\
\hline Cohen et al. & 2009 & 2,75 \\
\hline Gunka et al. & 2009 & 1,17 \\
\hline Dick et al. & 2010 & 0,73 \\
\hline Kurklinsky et al. & 2010 & 7,19 \\
\hline Venkatachalam et al. & 2011 & 1,17 \\
\hline Avgerinos et al. & 2013 & 2,20 \\
\hline da Cunha Júnior et al. & 2013 & 0,42 \\
\hline Grimm et al. & 2013 & 1,30 \\
\hline Bisoi et al. & 2014 & 1,17 \\
\hline Yavuz et al. & 2015 & 2,18 \\
\hline White et al. & 2016 & 3,24 \\
\hline Yadav et al. & 2016 & 0,44 \\
\hline Yun et al. & 2016 & 2,14 \\
\hline Zucker et al. & 2016 & 2,00 \\
\hline Al-Katib & 2017 & 3,24 \\
\hline Ananthan et al. & 2017 & 3,64 \\
\hline Bekci et al. & 2017 & 1,71 \\
\hline Hansraj et al. & 2017 & 0,85 \\
\hline White, Camerota & 2017 & 0,85 \\
\hline de Macedo & 2018 & 0,42 \\
\hline
\end{tabular}




\section{DESENVOLVIMENTO}

\section{Epidemiologia}

$\mathrm{O}$ primeiro paciente com síndrome de quebra-nozes foi identificado em 1950. Contudo, a compressão foi documentada por venografia duas décadas após ${ }^{15}$. A prevalência exata da síndrome de quebra-nozes é desconhecida devido à variabilidade dos sintomas e da ausência de diagnóstico precoce, mas tende a ser ligeiramente maior nas mulheres ${ }^{8,10-13}$. A idade dos pacientes pode variar da infância até a sétima década de vida. Contudo, a maioria dos pacientes sintomáticos está na segunda ou terceira década de vida, podendo ocorrer um segundo pico de síndrome de quebranozes em mulheres de meia-idade ${ }^{11}$. Embora haja relatos de casos que ocorreram entre irmãos, o fenômeno de quebra-nozes não é hereditário ${ }^{11}$. Segundo Okada et al., uma das causas mais importantes de hematúria bruta ou microscópica pode ser a síndrome do cassete, com uma prevalência relativamente alta em crianças de acordo com seus critérios ultrassonográficos ${ }^{16}$, indicando que essa síndrome pode ter sido subestimada no passado

\section{Fisiopatologia}

Os termos síndrome de quebra-nozes e fenômenos de nozes são muito usados de forma indistinta na literatura. Contudo, Shin e $\mathrm{Lee}^{17}$ destacam que a anatomia do fenômeno de quebranozes nem sempre está relacionado a sintomas clínicos, podendo representar uma variante anatômica normal ou ser explicado por outras condições. Dessa forma, o termo síndrome de quebra-nozes deve ser utilizado em casos de pacientes com sintomas clínicos típicos associados a características morfológicas sugestivas do fenômeno de nozes ${ }^{11,15,17}$. O mecanismo fisiopatológico da síndrome de quebra-nozes não é totalmente compreendido, mas várias teorias foram propostas sobre a etiologia subjacente para a predisposição anatômica, que incluem ptose renal esquerda, curso anormalmente alto de VRE, ramificação da AMS anormal da aorta ${ }^{7,12,18}$. A diminuição da quantidade de tecido adiposo perirrenal e pararrenal e a curva lordótica lombar proeminente causando anteriorização da aorta também estão envolvidos na patogênese da síndrome de quebra-nozes ${ }^{12}$. Uma angulação caudal aguda da MAS causando compressão extrínseca na VRE contra a aorta representa a característica mais comum da síndrome de quebranozes e também é denominada síndrome de quebra-nozes anterior. A compressão da terceira porção do duodeno pela AMS pode co-ocorrer com a síndrome de quebra-nozes anterior. A síndrome de quebra-nozes posterior ocorre quando a VRE cursa por trás da aorta, entre a aorta abdominal e os corpos vertebrais ${ }^{7,15}$. Uma VRE circum-aórtica também foi apontada como causa, com aprisionamento anterior e posterior de $\mathrm{VRE}^{19}$. Outros fatores que também causam compressão da veia renal esquerda são excesso de tecido fibroso na origem da AMS, tumores pancreáticos e retroperitoneais, linfadenopatia para-aórtica e artéria testicular abrangente ${ }^{11,20}$. A posição de pé, devido a proptose visceral e o estreitamento do ângulo aorto-sensorial, agrava a compressão extrínseca e induz a obstrução venosa da VRE9 . Esse fenômeno gera aumento da pressão venosa proximal, induzindo o desenvolvimento de varizes e caminhos colaterais. A compressão pela AMS causa aumento de até $3 \mathrm{mmHg}$ (normal $<1 \mathrm{mmHg}$ ) no gradiente de pressão entre a VRE e veia cava inferior, levando a ruptura do septo de paredes finas entre as veias pequenas e o sistema de coleta no fórnix renal, causando hematúria ${ }^{21}$.

\section{Manifestações clínicas}

A compressão da VRE leva a hipertensão venosa e ocasiona o desenvolvimento de varizes na pelve renal, tendo como resultantes hematúria e dor de flanco esquerdo. A hematúria é o mecanismo mais comumente relatado e provavelmente ocorre pela ruptura do septo de paredes finas secundárias a altas pressões venosas no sistema de coleta renal. A dor é o segundo sintoma mais comum, caracterizada por flanco ou dor abdominal inferior e é agravada pelo ato de sentar, caminhar ou andar em veículo vibratório. Também pode ser encontrada dor abdominal atípica ${ }^{7,22,23}$. Homens podem apresentar varicocele do lado esquerdo e mulheres podem apresentar sintomas de congestão pélvica, como dismenorreia, disúria, dispareunia, varizes vulvares e pélvicas ${ }^{24,25}$. Frequentemente, são observadas veias varicosas nas extremidades. A hematúria microscópica assintomática e a proteinúria ortostática podem ocorrer, sendo que a hematúria microscópica pode se agravar para uma situação macroscópica ${ }^{7,26}$, constituindo a principal razão para consulta com o urologista ${ }^{27,28}$. 


\section{Diagnóstico}

A síndrome de Quebra-Nozes apresenta difícil diagnóstico, muitas vezes atrasado e somente depois de se descartar todas as demais causas de hematúria e dor de flanco. É sugerida a utilização de cistoscopia, ultrassonografia, tomografia computadorizada e venografia renal para estabelecimento do diagnóstico ${ }^{22,29,30}$. A figura 1 ilustra a síndrome de quebra-nozes por meio de flebografia pré-procedimento. O ultrassom com Doppler da veia renal examina a velocidade do fluxo através da estenose, cuja velocidade sistólica máxima da área de compressão é quatro vezes a da veia não estenosada e tem sido utilizada para realizar o diagnóstico de estenose da veia renal, apresentando relatos de sensibilidade e especificidade de $69 \%$ a $90 \%$ e $89 \%$ a $100 \%$, respectivamente ${ }^{7,11}$. A venografia de ressonância magnética é útil particularmente no diagnóstico da síndrome da congestão pélvica relacionada à compressão da veia renal, havendo protocolos avançados de imagens que geram imagens de diagnóstico excelentes e conseguem mapear a direção do fluxo, tornando-se um importante estudo de diagnóstico não invasivo para pacientes com síndrome da congestão pélvica secundária a Síndrome de Quebra-Nozes ${ }^{7}$. Dick et al. utilizaram a técnica multifásica de ressonância magnética com venografia (RMV) para registrar dinamicamente as veias ovarianas em pacientes com síndrome da congestão pélvica e confirmaram a presença de refluxo $^{31}$. Venografia com pressão de contração adjuvante constitui uma avaliação anatômica e fisiológica importante para os pacientes. As pressões da VRE são mensuradas, e a compressão extrínseca da VRE ocasiona obstrução de saída, causando hipertensão da VRE, e um gradiente de pressão renocaval é registrado. Geralmente, há 1 mmHg ou menos de gradiente de pressão entre a veia renal periférica e a veia cava inferior ${ }^{23,32}$. É altamente sugestivo de uma significativa estenose da veia renal a presença de gradiente de pressão de retração renocaval maior que $2 \mathrm{~mm}$ de $\mathrm{Hg}^{22}$. A venografia retrógrada com determinação do gradiente de pressão renocaval é o estudo de padrão ouro para confirmação do diagnóstico da Síndrome de Quebra-Nozes ${ }^{7}$. A Figura 1 ilustra impressão da AMS sobre a VER por meio de flebografia préprocedimento $^{33}$.

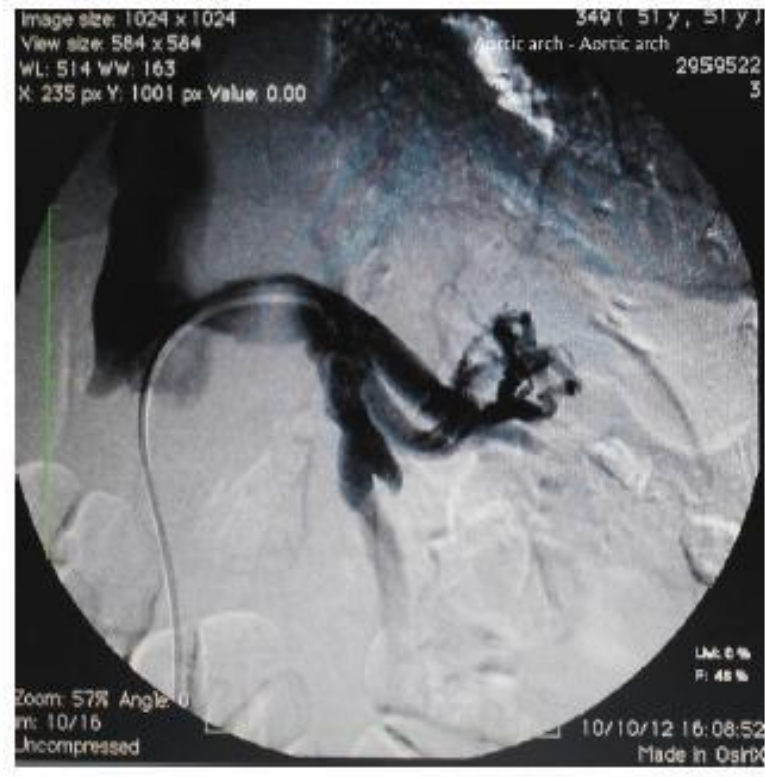

Figura 1. Fonte: Júnior-Cunha et al. (2013).

\section{Tratamento}

Entre os métodos cirúrgicos abertos, a transposição da VRE é a abordagem cirúrgica mais empregada e mais eficaz no tratamento da Síndrome de Quebra-Nozes anterior. A VRE é transposta caudalmente na veia cava inferior, restabelecendo sua trajetória distal ao ângulo aortomesentérico ${ }^{7}$. Atualmente, as técnicas para a reconstrução vascular aberta concentram-se na transposição da veia renal esquerda e autotransplante renal ${ }^{34-36}$. Shokeir et al. realizou o autoimplante do rim esquerdo, obtendo resultados satisfatórios ${ }^{37}$. Outras modalidades cirúrgicas descritas incluem a derivação da VRE com interposição de enxerto de politetrafluoretileno ${ }^{14}$; transposição de veia gonadal para veia cava inferior; bypass renocaval com veia safena ${ }^{7} \mathrm{e}$ transposição da veia ovariana esquerda para veia ilíaca externa esquerda ${ }^{38}$. A transposição da origem da AMS, reimplantando-a na aorta inferior à veia renal esquerda, é descrita como uma alternativa aos vários tipos de manipulação intervencionista ou cirúrgica da VRE ${ }^{39}$. Neste et al, em 1996, realizou pela primeira vez o manejo endovascular para compressão da VRE anterior, por meio de implante de stent endovascular percutâneo da $\mathrm{VRE}^{4,38}$. Cada vez mais, a abordagem endovascular para o manejo da síndrome de quebra-nozes tem sido considerada como alternativa viável ao tratamento cirúrgico aberto $^{7,40-42}$. Vários stents tem sido utilizados com bom sucesso técnico e resolução a longo prazo dos sintomas, como stent espiral Z-stent, o stent Niki, o stent Palmaz e o stent Smart Control ${ }^{4,43-45}$. 
Algumas complicações associadas ao manejo endovascular foram relatadas, tais como trombose, reestenose e o deslocamento e a migração do stent da VRE, podendo resultar em recorrência dos sintomas ${ }^{43,44,46}$.

\section{CONCLUSÃO}

A síndrome do quebra-nozes é uma condição incomum que ocorre devido à compressão extrínseca da VRE entre a aorta e a AMS. Possui manifestações diversas, sendo as mais comuns dores no flanco esquerdo e hematúria. O diagnóstico é difícil e, geralmente, tardio. Existem várias opções de tratamento, sendo o procedimento operatório aberto o mais realizado, e, destes, a transposição VRE tem sido a cirurgia mais empregada e mais eficaz no tratamento da síndrome. Uma alternativa menos invasiva ao tratamento cirúrgico aberto que vem ganhando espaço é a abordagem endovascular, apesar de algumas complicações terem sido relatadas.

\section{REFERÊNCIAS}

1. Hansraj N, Hamdi A, Khalifeh A, Wise E, Sarkar R, Toursavadkohi S. Nutcracker Syndrome: Case Report on the Management of Recurrent Stenosis after Stenting. Vasc Endovascular Surg. 2017. https://doi.org/10.1177/1538574417700012

2. Ananthan K, Onida S, Davies AH. Nutcracker Syndrome: An Update on Current Diagnostic Criteria and Management Guidelines. Eur J Vasc Endovasc Surg. 2017;53(6):886-94. http://doi.org/10.1016/j.ejvs.2017.02.015

3. Chait A, Matasar KW, Fabian CE, et al. Vascular impressions on the ureters. Am J Roentgenol RadiumTher Nucl Med. 1971;111:729-49. https://doi.org/10.2214/ajr.111.4.729

4. Venkatachalam S, Bumpus K, Kapadia SR, Gray B, Lyden S, Shishehbor MH. The nutcracker syndrome. Ann Vasc Surg. 2011;25(8):1154-64. http://dx.doi.org/10.1016/j.avsg.2011.01.002

5. Al-Katib S, Shetty M, Jafri SMA, Jafri SZH. Radiologic Assessment of Native Renal Vasculature: A Multimodality Review. Radiographics. 2017;37(1):136-56. https://doi.org/10.1148/rg.2017160060

6. Bekci T, Ceyhan Bilgici M, Genc G, Tekcan D, Veysel Polat A, Tomak L. Evaluation of Renal Parenchyma Elasticity With Acoustic Radiation Force Impulse Quantification in Nutcracker Syndrome and Comparisons With Grayscale Doppler Sonography and Laboratory Findings. J
Ultrasound $\quad$ Med. 2017;36(1):61-7. http://doi.wiley.com/10.7863/ultra.16.03010

7. White JM, Comerota AJ. Venous Compression Syndromes. Vasc Endovascular Surg. 2017;51(3):155-68.

https://doi.org/10.1177\%2F1538574417697208

8. Yadav P, Lal H, Verma P, Mourya C. Vascular compression of left renal vein: The nutcracker phenomenon. BMJ Case Rep. 2016; 2016:1-2. http://dx.doi.org/10.1136/bcr-2016-218134

9. Grimm LJ, Engstrom BI, Nelson RC, Kim CY. Incidental detection of nutcracker phenomenon on multidetector ct in an asymptomatic population: Prevalence and associated findings. J Comput Assist Tomogr. 2013;37(3):415-8.

10. Yun SJ, Lee JM, Nam DH, Ryu JK, Lee SH. Discriminating renal nutcracker syndrome from asymptomatic nutcracker phenomenon using multidetector computed tomography. Abdom Radiol. 2016;41(8):1580-8. https://doi.org/10.1007/s00261-016-0717-8

11. Kurklinsky AK, Rooke TW. Nutcracker Phenomenon and Nutcracker Syndrome. Mayo Clin Proc [Internet]. 2010;85(6):552-9. http://dx.doi.org/10.4065/mcp.2009.0586

12. Bisoi AK, Sahu MK, Chander C N, Agarwala $\mathrm{S}$, Chauhan S. Nutcracker syndrome in a young female with solitary functional left ovary: A surgical challenge. Ann Vasc Surg. 2014;28(8):1938.e1-1938.e3.

http://dx.doi.org/10.1016/j.avsg.2014.07.019

13. da Cunha Júnior JR, de Souza TC, Feitosa AT, Brizzi JR, Tinoco JA. Tratamento endovascular da síndrome de Quebra-nozes (Nutcracker). J Vasc Bras. 2013;12(3):247-51. http://dx.doi.org/10.1590/jvb.2013.034

14. Rudloff U, Holmes RJ, Prem JT, Faust GR, Moldwin R, Siegel D. Mesoaortic compression of the left renal vein (nutcracker syndrome): Case reports and review of the literature. Ann Vasc Surg. 2006;20(1):120-9.

https://doi.org/10.1007/s10016-005-5016-8

15. Avgerinos ED, McEnaney R, Chaer RA. Surgical and endovascular interventions for nutcracker syndrome. Semin Vasc Surg [Internet]. 2013;26(4):170-7.

http://dx.doi.org/10.1053/j.semvascsurg.2014.06.0 $\underline{14}$

16. Shin JI, Lee JS, Kim MJ. The Prevalence, Physical Characteristics and Diagnosis of Nutcracker Syndrome. Eur J Vasc Endovasc Surg. 2006;32(3):335-6.

https://doi.org/10.1016/j.ejvs.2006.04.030 
17. Shin J Il, Park JM, Lee JS, Kim DH, Jeong HJ. Development of Graves' disease during cyclosporin treatment for severe HenochSchönlein nephritis [6]. Nephrol Dial Transplant. 2005;20(9):2014-5.

\section{https://doi.org/10.1093/ndt/gfh959}

18. Yavuz S, Kiyak A, Er A, Korkmaz O. The utility of bioimpedance analysis for monitoring the children with nutcracker syndrome. Eur J Pediatr. 2015;174(10):1393-7.

https://doi.org/10.1007/s00431-015-2535-3

19. Cohen F, Amabile P, Varoquaux A, Piquet P, Bartoli JM, Vidal V, et al. Endovascular Treatment of Circumaortic Nutcracker Syndrome. J Vasc Interv Radio. 2009;20(9):1255-7. http://dx.doi.org/10.1016/j.jvir.2009.05.022

20. Neste MG, Belcher KK. Case Report Endovascular Stent Placement as a Treatment for Renal Venous Hipertension. J Vasc Interv Radiol. 1996;5-7.

https://doi.org/10.1016/j.clineuro.2009.10.018 21. Ahmed K, Sampath R, Khan MS. Current trends in the diagnosis and management of renal nutcracker syndrome: A review. Eur J Vasc Endovasc Surg. 2006;31(4):410-6. https://doi.org/10.1016/j.ejvs.2005.05.045

22. Gunka I, Navratil P, Lesko M, Jiska S, Raupach J. Laparoscopic Left Renal Vein Transposition for Nutcracker Syndrome. Ann Vasc Surg [Internet]. 2016;31(2):209.e1-209.e5.

http://dx.doi.org/10.1016/j.jvs.2008.09.051

23. Nishimura, Yasumasa; Fushiki, Masato; Yoshida, Mitsunori; Nakamura, Keiya; Imai, Masao; Ono, Tetsuo; Morikawa, Susumu; Hatayama, Tadashi; Komatz Y. Left Renal Vein Hypertension in Patients with Left Renal Bleeding of Unknown Origin. Radiology. 1986;160:663-7. https://doi.org/10.1148/radiology.160.3.3737903

24. Scultetus AH, Villavicencio JL, Gillespie DL. The nutcracker syndrome: Its role in the pelvic venous disorders. J Vasc Surg. 2001;34(5):812-9. https://doi.org/10.1067/mva.2001.118802

25. Zerhouni EA, Siegelman SS, Walsh PC, White RI. Elevated pressure in the left renal vein in patients with varicocele: preliminary observations. J Urol [Internet]. 1980;123(4):512-3. https://doi.org/10.1016/s0022-5347(17)55996-0

26. Correia T, Cardoso A, Soares R, et al. Síndrome de Quebra-nozes: A propósito de um caso clínico. Acta Urologica. 2007;24(4):49-52.

27. Hohenfelliner M, Steinbach F, Schultz-Lampel D, Schantzen W, Walter K, Cramer BM, et al. The Nutcracker Syndrome: New Aspects of Pathophysiology, Diagnosis and Treatment. Vol.
146, The Journal of Urology. 1991. https://doi.org/10.1016/s0022-5347(17)37893-x

28. Wendel RG, Crawford ED, Hehman KN. The "nutcracker" phenomenon: an unusual cause for renal varicosities with hematuria. J Urol [Internet]. 1980;123(5):761-3.

https://doi.org/10.1016/S0022-5347(17)56121-2 29. Stavros AT, Sickler KJ, Menter RR. Color duplex sonography of the nutcracker syndrome (aortomesenteric left renal vein compression). J Ultrasound Med. 1994;13(7):569-74. https://doi.org/10.7863/jum.1994.13.7.569

30. Zucker EJ, Ganguli S, Ghoshhajra BB, Gupta $\mathrm{R}$, Prabhakar AM. Imaging of venous compression syndromes. Cardiovasc Diagn Ther. 2016;6(6):519-32.

http://dx.doi.org/10.21037/cdt.2016.11.19

31. Dick EA, Burnett C, Anstee A, Hamady M, Black D, Gedroyc WMW. Timeresolved imaging of contrast kinetics three-dimensional (3D) magnetic resonance venography in patients with pelvic congestion syndrome. $\mathrm{Br} \mathrm{J}$ Radiol. 2010;83(994):882-7.

https://dx.doi.org/10.1259\%2Fbjr\%2F82417499

32. Beinart C, Sniderman KW, Tamura S, Vaughan $\mathrm{ED}$, Sos TA. Left renal vein to inferior vena cava pressure relationship in humans. $\mathbf{J}$ Urol. 1982;127(6):1070-1.

https://doi.org/10.1016/s0022-5347(17)54230-5

33. Cunha Junior Jorge Ribeiro da, Souza Tiago Coutas de, Feitosa Adilson Toro, Brizzi Jose Ricardo, Tinoco Juliana Amaral. Tratamento endovascular da Sindrome de Quebra-nozes (Nutcracker). J. vasc. bras. 2013;12(3): 247-251. http://dx.doi.org/10.1590/jvb.2013.034

34. Hohenfellner M, D'Elia G, Hampel C, Dahms $S$, Thüroff JW. Transposition of the left renal vein for treatment of the nutcracker phenomenon: Longterm follow-up. Urology. 2002;59(3):354-7. https://doi.org/10.1016/j.avsg.2014.07.022

35. Stewart BH, Reiman G. " NUTCRACKER" SYNDROME Managed by Direct Renocaval Reimplantation. 1982;XX(4). Available from: https://doi.org/10.1016/0090-4295(82)90457-5.

36. Chuang CK, Chu SH, Lai PC. The nutcracker syndrome managed by autotransplantation. J Urol. 1997;157(5):1833-4.

https://doi.org/10.1016/S0022-5347(01)64872-9 37. Shokeir El-diasty AT, Ghoneim M. The nutcracker syndrome: new methods of diagnosis and treatment. $\mathrm{Br}$ Journal of Urology. 1994;74:139-43. https://doi.org/10.1111/j.1464410X.1994.tb16574.X 
38. White J V., Ryjewski C, Messersmith RN, Sbrana F, Schwartz LB. Left ovarian to left external iliac vein transposition for the treatment of nutcracker syndrome. J Vasc Surg. 2016;4(1):1148. http://dx.doi.org/10.1016/j.jvsv.2015.10.003 39. de Macedo GL, Dos Santos MA, Sarris AB, Gomes RZ. Diagnosis and treatment of the Nutcracker syndrome: a review of the last 10 years. J Vasc Bras. 2018 ;17(3):220-228. https://doi.org/10.1590/1677-5449.012417

40. Park YB, Lim SH, Ahn JH, Kang E, Myung SC, Shim HJ, et al. Nutcracker syndrome: Intravascular stenting approach. Nephrol Dial Transplant. 2000;15(1):99-101.

https://doi.org/10.1093/ndt/15.1.99

41. Hosotani Y, Kiyomoto H, Fujioka H, Takahashi N, Kohno M. The nutcracker phenomenon accompanied by renin-dependent hypertension [1]. Am J Med. 2003;114(7):617-8. https://doi.org/10.1016/s0002-9343(03)00091-3

42. Zhou MIN. Case Reports. Intravenous stent placement for treatment of the nutcracker syndrome. 2003;170:1934-5. Available from: https://doi.org/10.1097/01.ju.0000089872.39883.a $\underline{3}$

43. Hartung O, Grisoli D, Boufi M, Marani I, Hakam Z, Barthelemy P, et al. Endovascular stenting in the treatment of pelvic vein congestion caused by nutcracker syndrome: Lessons learned from the first five cases. J Vasc Surg. 2005;42(2):275-80.

https://doi.org/10.1016/j.jvs.2005.03.052

44. Kim SJ, Kim CW, Kim S, Lee TH, Lee JW, Lee $\mathrm{SH}$, et al. Long-term follow-up after endovascular stent placement for treatment of nutcracker syndrome. J Vasc Interv Radiol. 2005;16(3):42831.

https://doi.org/10.1097/01.rvi.0000149904.26242. $\underline{54}$ 45. Zhang H, Li M, Jin W, San P, Xu P, Pan S. The Left Renal Entrapment Syndrome: Diagnosis and Treatment. Ann Vasc Surg. 2007;21(2):198-203. https://doi.org/10.1016/j.avsg.2006.10.021

46. Chen S, Zhang H, Tian L, Li M, Zhou M, Wang Z. A stranger in the heart: LRV stent migration. Int Urol Nephrol. 2009;41(2):427-30. https://doi.org/10.1007/s11255-008-9478-9 